\title{
A simple and novel control strategy for semi-active vibration suppression by a magnetorheological damper
}

\author{
Authors \\ Shuto NAGAMATSU* and Toshihiko SHIRAISHI (*corresponding author) \\ E-mail \\ shutonagamatsu0617@gmail.com; nagamatsu-shuto-df@ynu.jp (Shuto Nagamatsu), \\ shira@ynu.ac.jp (Toshihiko Shiraishi)
}

\begin{abstract}
Affiliation
Yokohama National University Graduate School of Environment and Information Sciences

Faculty of Environment and Information Sciences, Artificial Environment

(79-7 Tokiwadai, Hodogaya, Yokohama, 240-8501, Japan)
\end{abstract}

\begin{abstract}
Conventional skyhook-based ON-OFF control switches the damping force on a vibration suppression target according to the sign of the product of the target and relative velocities (which is called the condition function). Here, we propose a control strategy that uses a novel condition function for improved performance. The proposed strategy is formulated based on the theory of forced vibration with base excitation. Its effect upon semi-active vibration performance is investigated via numerical simulations and experimental tests of the vibration suppression of a small structure equipped with a magnetorheological (MR) damper. In the simulations, the proposed control strategy can offer high-performance semi-active vibration suppression, even in the presence of force delays in the damper. The experiments show that the displacement response with the proposed control is lower than that with the conventional skyhook-based control over the entire frequency range; furthermore, the desired performance can be achieved when the proposed condition function is used with velocity-proportional control. The simplicity and high performance demonstrated by the proposed control strategy make it applicable to semi-active vibration suppression of practical systems, even in the presence of unavoidable force delays in controllable dampers.
\end{abstract}

\section{Keywords}

Semi-active vibration suppression, Single-degree-of-freedom, Condition function, ON-OFF control, Skyhook damper scheme, MR damper 
1. Introduction

Semi-active vibration suppression is a method of suppressing vibration by controlling passive devices with variable damping or stiffness according to the vibration state. By appropriately designing the control strategy, the dynamic characteristics of the system can be varied as intended without using active devices driven by eternal power; thus, a high performance can be achieved with a small energy consumption. To successfully suppress the vibration of a single-degree-of-freedom system, a damper must act as a passive ON state around the natural frequency and as a passive OFF state in the high-frequency range. Unlike passive states that cannot achieve high vibration suppression performance over a wide frequency range because of their constant damping characteristics, semi-active methods theoretically can achieve it by varying the input signal from the controller to the damper according to the excitation and/or responses measured by the sensors (Casciati et al., 2012; Saaed et al., 2013; Symas and Constantinou, 1999).

One of the most successful active control strategies is the skyhook damper scheme proposed for vehicle suspensions (Karnopp et al., 1974); it has been demonstrated that vibration can be suppressed over the entire frequency range through the exertion of a damping force to reduce the absolute velocity of the suppression target. The desired damping force, $F_{d}$, is expressed as

$$
F_{d}=-c_{\text {sky }} \dot{x}
$$

where $c_{\text {sky }}$ and $\dot{x}$ denote the proportional gain and the absolute velocity of the target, respectively (Figure 1). During semi-active vibration suppression by a damper installed between the target and the base, the damper generates a force proportional to the relative velocity $\dot{x}-\dot{x}_{0}$, where $\dot{x}_{0}$ is the base velocity; thus, the desired force $F_{d}$ cannot be completely achieved. Under these constraints, the damping force is switched according to the following simple equation, which emulates the skyhook damper scheme:

$$
F= \begin{cases}F_{O N}, & \dot{x}\left(\dot{x}-\dot{x}_{0}\right)>0 \\ F_{O F F}, & \dot{x}\left(\dot{x}-\dot{x}_{0}\right) \leq 0 .\end{cases}
$$

This equation switches between large damping forces in the ON state $\left(F_{O N}\right)$ and small ones in the OFF state $\left(F_{O F F}\right)$ according to the sign of $\dot{x}\left(\dot{x}-\dot{x}_{0}\right)$, which is called the condition function (Liu et al., 2005). This switching allows the damper to generate large forces only when the absolute and relative velocities are in the same direction. When they are opposed, the damping force is minimized so as not to increase the absolute velocity (Figure 2). When $F_{O N}$ is provided by variable signals, the continuous skyhook-based control is achieved to exert forces with the required phase and amplitude. Conversely, to ensure that the force is exerted in the desired direction, when $F_{O N}$ is provided by a single signal, ON-OFF skyhook-based control is achieved. Comparing these two control schemes, ON-OFF control benefits from a simpler implementation (Liu et al., 2005). The desired performance of the conventional $\mathrm{ON}-\mathrm{OFF}$ control is obtained as the relationship between the transmissibility and the frequency ratio. The transmissibility is defined as the amplitude ratio of the target displacement to the base one, $|x| /\left|x_{0}\right|$, whereas the frequency ratio is defined as the ratio of the excitation frequency to the natural frequency, $\omega / \omega_{n}$. When the damping ratios of 1.0 and 0.1 are switched according to the sign of $\dot{x}\left(\dot{x}-\dot{x}_{0}\right)$ in the simulation, transmissibility is successfully reduced over the entire frequency range (Figure 3).

However, in experiments, the vibration suppression performance of such conventional ON-OFF control is 
degraded because of the delay in the control system and especially that in the force of the damper (Cha et al., 2013; Qin et al., 2017; Strecker et al., 2015a; Strecker et al., 2015b; Strecker et al., 2018). The delay in switching from ON to OFF when $\dot{x}\left(\dot{x}-\dot{x}_{0}\right)$ changes from positive to negative induces a degradation of performance, since the large damping force is exerted in the opposite direction to that intended. Furthermore, because of the delay in switching from OFF to ON when $\dot{x}\left(\dot{x}-\dot{x}_{0}\right)$ changes from negative to positive, the large force required cannot be exerted to reduce the absolute velocity.

Magnetorheological (MR) dampers have been widely studied as controllable devices with high responses (Dyke et al., 1996; Dyke et al., 1998; Yang et al., 2002; Yao et al., 2002); these are controllable dampers using an MR fluid composed of micron-sized ferromagnetic particles and base oils. The rheological characteristics of MR fluids can be varied instantaneously and substantially through the application of an external magnetic field. An electric current is converted into a magnetic field by the mounted electromagnet; thus, the damping force of the MR dampers can be controlled by the supplied currents. The response times of the MR dampers have been reduced by designing the entire system considering several factors affecting the delay, including the physical dimensions of the damper, the method of driving the electronics, and the response time of the MR fluid. However, since it is difficult for a single system to simultaneously satisfy the requirements for physical dimension, electronics, and MR fluid, the issue of force delays has not been completely overcome. Previous studies have reported that the response times of the MR dampers are typically several tens of milliseconds (Cha et al., 2013; Strecker et al., 2015b). Thus, control strategies that can provide high performance, even under delays of several tens of milliseconds in the damper, should be proposed to successfully suppress vibrations during experiments.

In this study, to achieve high vibration suppression performance in the presence of force delays, control strategies using a novel condition function are proposed and formulated; furthermore, the advantage of the proposed control strategy over the conventional one is demonstrated through numerical simulations and experimental tests of the vibration suppression of a small single-degree-of-freedom model structure equipped with an MR damper.

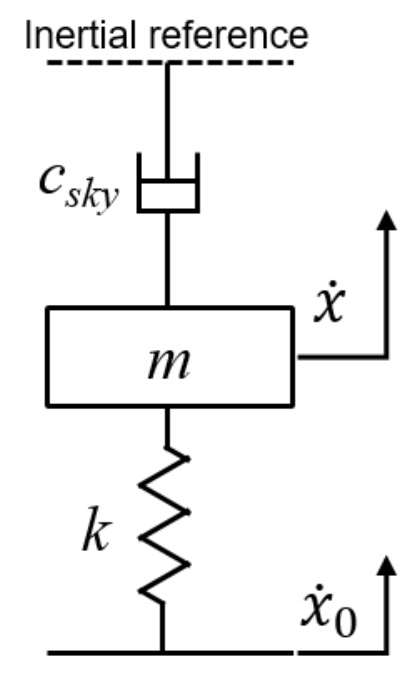

Figure 1. Concept of the conventional skyhook damper scheme. 


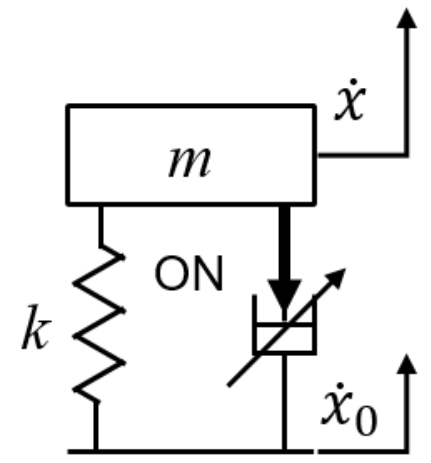

(a)

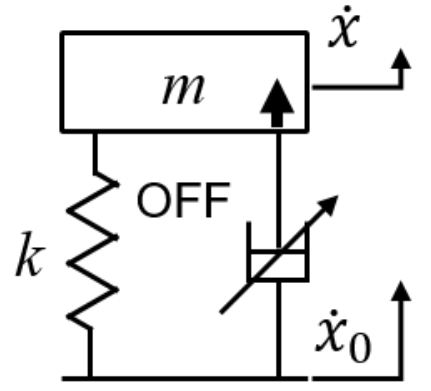

(b)

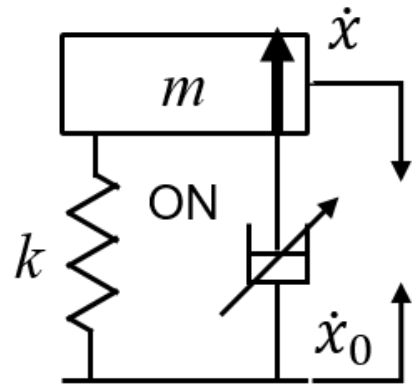

(c)

Figure 2. Emulation of the conventional skyhook damper scheme by ON/OFF switching according to Eq. (2): (a) $\dot{x}>$ 0 and $\dot{x}-\dot{x}_{0}>0$; (b) $\dot{x}>0$ and $\dot{x}-\dot{x}_{0}<0$; (c) $\dot{x}<0$ and $\dot{x}-\dot{x}_{0}<0$. Thick arrows indicate the damping forces.

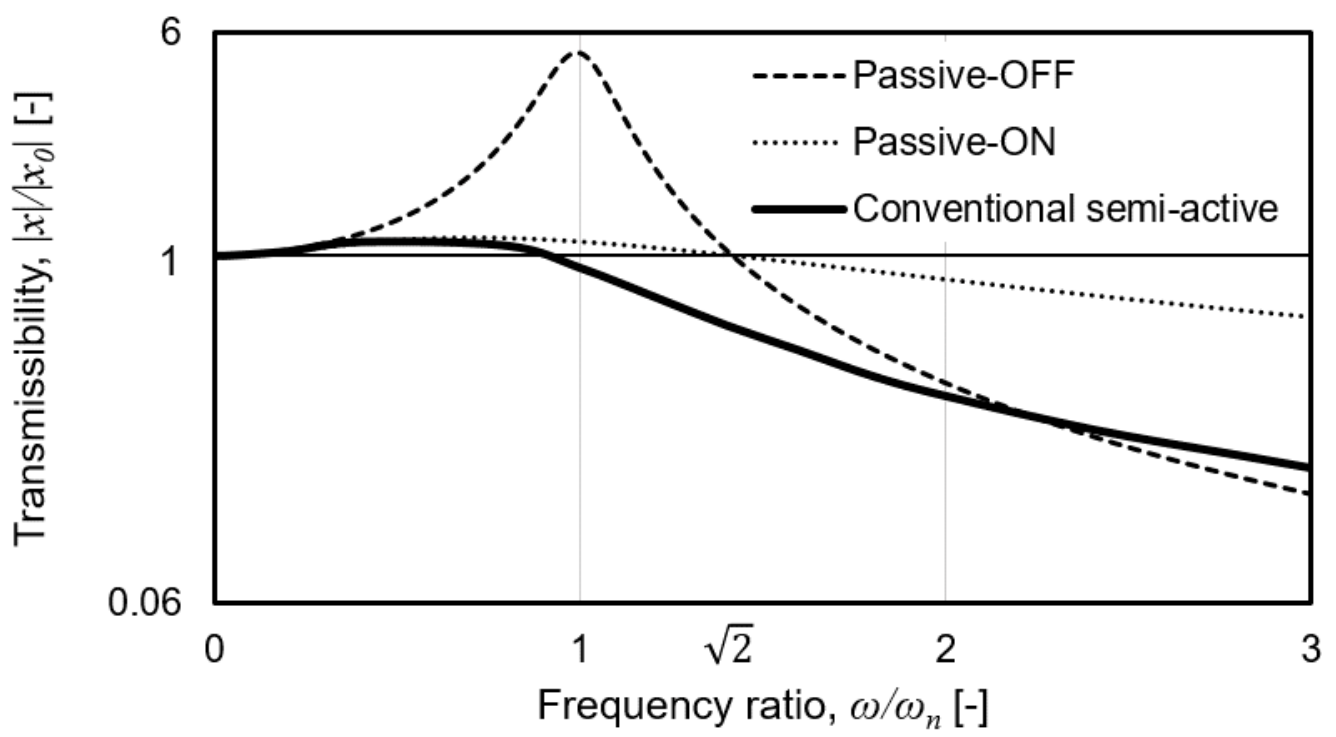

Figure 3. Desired vibration suppression performance of the conventional skyhook-based ON-OFF control obtained by simulation. The damping ratios of 1.0 and 0.1 are switched according to the sign of $\dot{x}\left(\dot{x}-\dot{x}_{0}\right)$. 
2. Proposed Control Strategy

To achieve a high vibration suppression performance even in the presence of force delays, a simple and novel control strategy is proposed in this study. It is expressed by the following equation:

$$
F= \begin{cases}F_{O N}, & \dot{x}\left(\dot{x}+\dot{x}_{0}\right)>0 \\ F_{O F F}, & \dot{x}\left(\dot{x}+\dot{x}_{0}\right) \leq 0 .\end{cases}
$$

Eq. (3) that can determine the input signal to the damper by only the target and base velocities is as simple as the abovementioned skyhook-based control. Furthermore, since Eq. (3) does not require the model and parameter adjustment, it is much simpler than previous model-based controls (Qin et al., 2017; Yoon et al., 2021) and PID controls (Choi et al., 2016).

Here, the conventional condition function $\dot{x}\left(\dot{x}-\dot{x}_{0}\right)$ is replaced by $\dot{x}\left(\dot{x}+\dot{x}_{0}\right)$. This simple modification offers a completely different approach to the suppression of vibrations from single-degree-of-freedom systems. The feasibility of this strategy for semi-active vibration suppression can be demonstrated based on the theory of forced vibration with base excitation. Considering the theoretical frequency response of the velocity-amplitude ratio $V$ and the phase difference under base excitation (Figure 4), the range of frequency ratios can be divided into three parts: $0<\omega / \omega_{n}<1,1 \leq \omega / \omega_{n} \leq \sqrt{2}$, and $\omega / \omega_{n}>\sqrt{2}$. The sign of $\dot{x}\left(\dot{x}+\dot{x}_{0}\right)$ can be calculated at each range, assuming that the damping ratio of the target system is low. This assumption is valid for practical applications, since the general damping ratios for structures or vehicle suspensions are low enough that the phase difference can be approximated as $0^{\circ}$ at $0<\omega / \omega_{n}<1$ and $180^{\circ}$ at $\omega / \omega_{n} \geq 1$. Expressing the base velocity $\dot{x}_{0}$ as $v_{0} \sin \omega t$, the target velocity $\dot{x}$ can be expressed as follows:

$$
\dot{x}=\left\{\begin{array}{lr}
+\left|\frac{v}{v_{0}}\right| \dot{x}_{0}=+V v_{0} \sin \omega t, & 0<\omega / \omega_{n}<1, \\
-\left|\frac{v}{v_{0}}\right| \dot{x}_{0}=-V v_{0} \sin \omega t, & 1<\omega / \omega_{n},
\end{array}\right.
$$

where $v$ denotes the amplitude of the target velocity. The sign of $\dot{x}\left(\dot{x}+\dot{x}_{0}\right)$ is calculated using these simple velocity expressions.

The calculation results demonstrate that $\dot{x}\left(\dot{x}+\dot{x}_{0}\right)$ is 0 or more in $\omega / \omega_{n} \leq \sqrt{2}$, whereas it is 0 or less in $\omega / \omega_{n}>\sqrt{2}$ (Table 1); therefore, applying Eq. (3) for the switching of semi-active vibration suppression allows the damper to act in the ON state when $\omega / \omega_{n} \leq \sqrt{2}$ and in the OFF state when $\omega / \omega_{n}>\sqrt{2}$ (Figure 5). This approach is completely different from the skyhook damper scheme that always dissipates energy during the excitation. It is interpreted from the viewpoint of ON/OFF switching at $\omega / \omega_{n}=\sqrt{2}$ on the frequency response instead of energy transfer and energy dissipation. In terms of physical meaning, the damper is switched to the ON state when the target and base velocities are in the same direction (Figure 6 (a)). The ON state is also provided when the absolute value of the target velocity is greater than the base one, even if the velocities are in opposite directions (Figure 6 (b)). Conversely, the damper is switched to the OFF state when the velocities are in opposite directions and the absolute value of the base velocity is greater than that of the target (Figure 6 (c)).

This strategy is based on the theory of forced vibration, as opposed to the conventional strategy, which follows the desired damping force required in the skyhook damper scheme; thus, the performance of the proposed control can be maintained even in the presence of force delays. Furthermore, the response with the proposed control can be reduced to the same level as that of the OFF state in the high-frequency range; this theoretically cannot be 


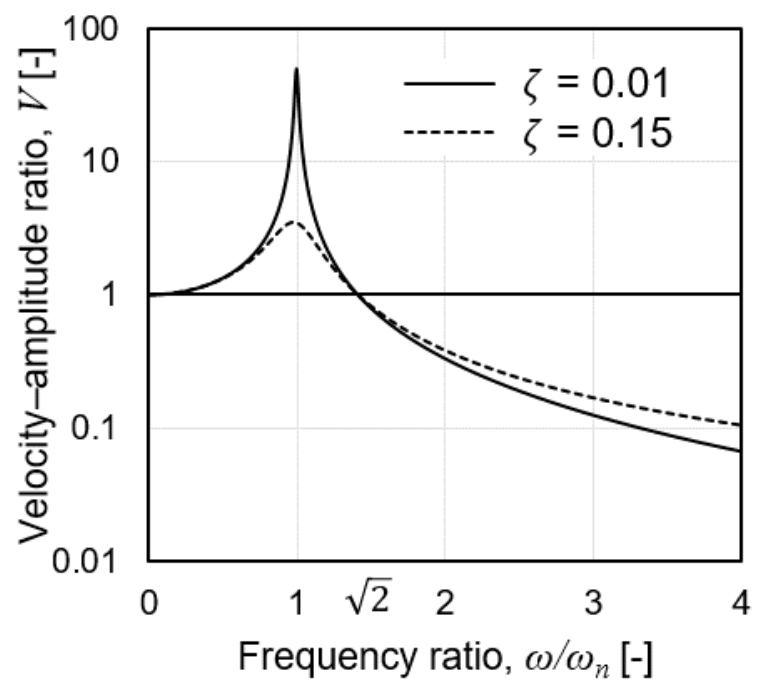

(a)

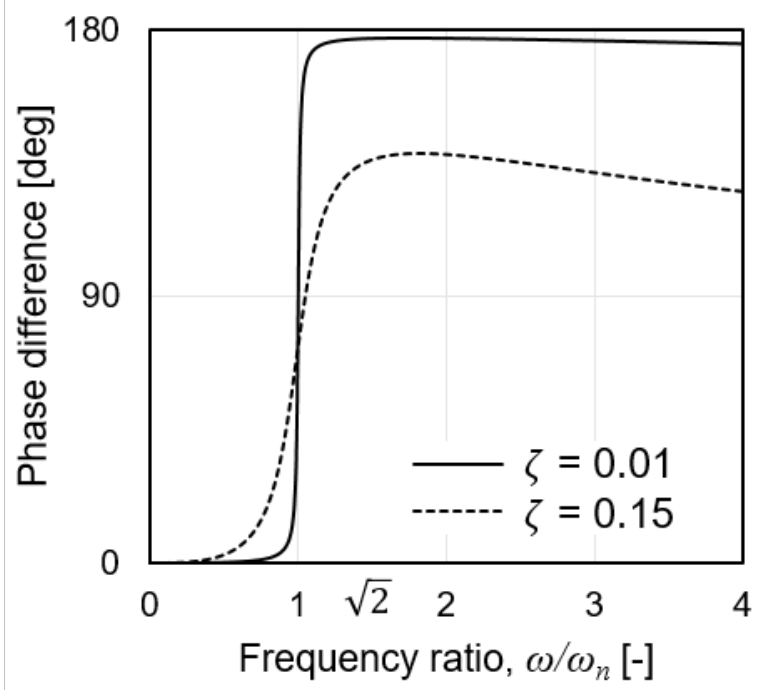

(b)

Figure 4. Theoretical frequency responses of (a) the velocity-amplitude ratio and (b) the phase difference at damping ratios of 0.01 and 0.15 .

Table 1. Damping forces provided by the proposed control strategy using $\dot{x}\left(\dot{x}+\dot{x}_{0}\right)$. The calculation is performed by expressing $\dot{x}_{0}$ as $v_{0} \sin \omega t$.

\begin{tabular}{cccc}
\hline Frequency ratio [-] & $0<\omega / \omega_{n}<1$ & $1 \leq \omega / \omega_{n} \leq \sqrt{2}$ & $\sqrt{2}<\omega / \omega_{n}$ \\
\hline Base velocity, $\dot{x}_{0}$ & $v_{0} \sin \omega t$ & $v_{0} \sin \omega t$ & $v_{0} \sin \omega t$ \\
Target velocity, $\dot{x}$ & $+V v_{0} \sin \omega t$ & $-V v_{0} \sin \omega t$ & $-V v_{0} \sin \omega t$ \\
Amplitude ratio $[-]$ & $V \geq 1$ & $V \geq 1$ & $0<V<1$ \\
$\dot{x}\left(\dot{x}+\dot{x}_{0}\right)$ & $V(V+1) v_{0}^{2} \sin ^{2} \omega t$ & $V(V-1) v_{0}^{2} \sin ^{2} \omega t$ & $V(V-1) v_{0}^{2} \sin ^{2} \omega t$ \\
Sign of $\dot{x}\left(\dot{x}+\dot{x}_{0}\right)$ & 0 or more & 0 or more & 0 or less \\
Force by Eq. (3) & $F_{O N}$ & $F_{O N}$ & $F_{O F F}$ \\
\hline
\end{tabular}




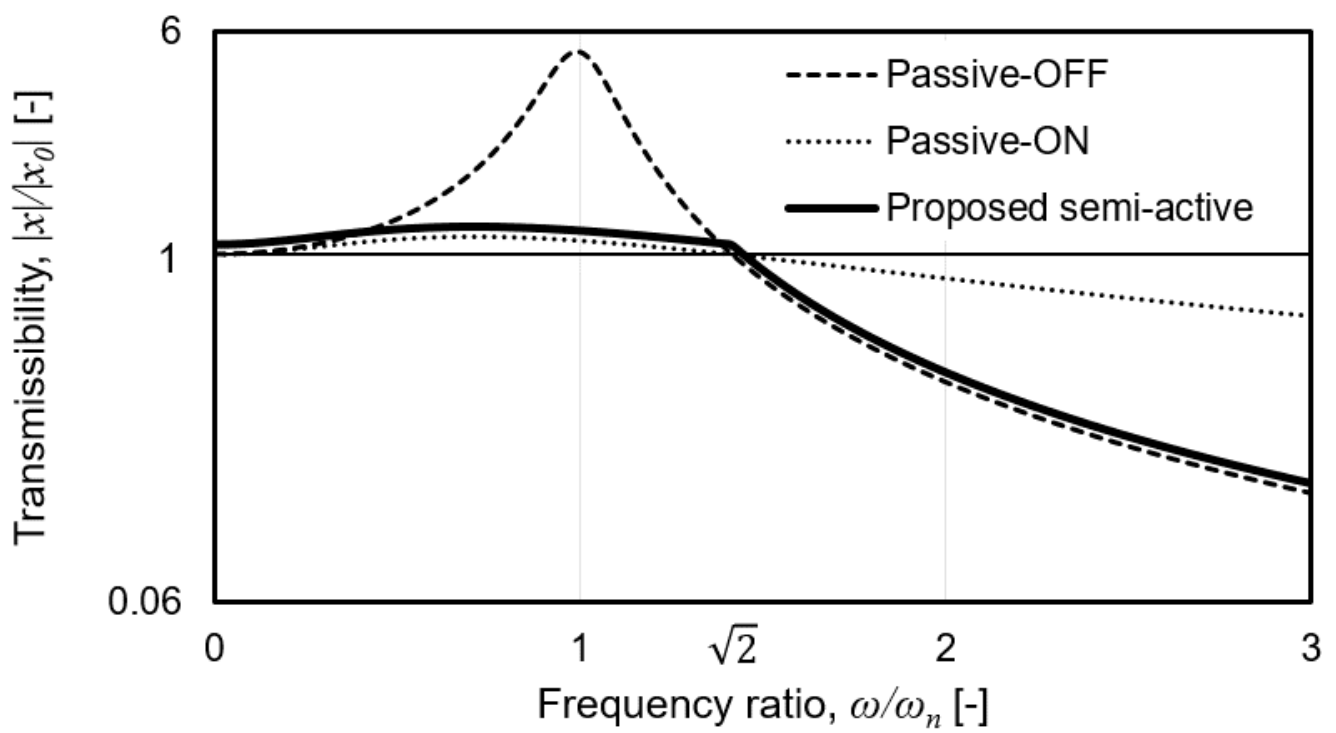

Figure 5. Desired performance of the proposed control strategy using $\dot{x}\left(\dot{x}+\dot{x}_{0}\right)$ as the condition function.

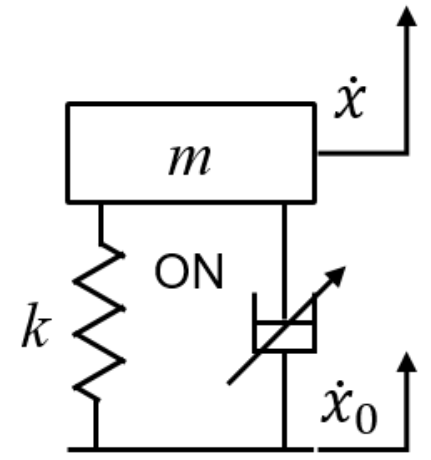

(a)

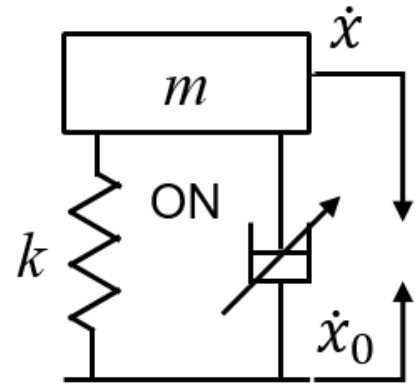

(b)

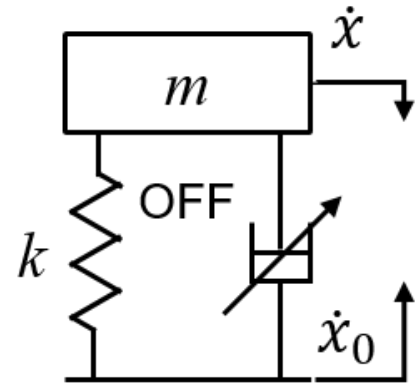

(c)

Figure 6. Relationship between the ON/OFF switching by Eq. (3) and the target and base velocities in the ranges of (a) $0<\omega / \omega_{n}<1$, (b) $1 \leq \omega / \omega_{n} \leq \sqrt{2}$, and (c) $\sqrt{2}<\omega / \omega_{n}$. 
3. Methods

To investigate the effect of the proposed control strategy upon the semi-active vibration suppression performance, numerical simulations and experimental tests of vibration suppression in a structure equipped with an MR damper were conducted. As a performance indicator, we evaluated the reduction in the absolute displacement of the upper floor of the structure.

\subsection{Target structure}

The target of vibration suppression was a small single-degree-of-freedom model structure with a height of 400 mm, equipped with an MR damper (Figure 7). To construct a structure with a low damping ratio, the damper must generate small damping forces in the OFF state. A shear-type damper using magnetorheological grease (Shiraishi and Sugiyama, 2015) was used as an appropriate device.

The shear-type MR grease damper was an excellent device, which solved two issues in conventional pressuretype MR fluid dampers. One was the sedimentation of their magnetic particles caused by the density mismatch between the magnetic particles and their carrier oil, resulting in performance degradation. The other was the low dynamic range due to the sliding friction force acting on the sealing elements of dampers, where the dynamic range was the ratio of total force to the uncontrollable force. They were overcome by the MR grease with high dispersion stability and the shear-type damper without the need for sealing elements, respectively. In practical application, whereas an MR fluid cannot be used in a damper without sealing elements since it may leak out during operation, the semi-solid MR grease can be applied to it without leakage. Furthermore, the MR grease, which allowed the sealing elements to be removed, offered very small damping forces in the OFF state and high dynamic range despite its drawback of higher viscosity than that of MR fluids. A low damping ratio of approximately 0.15 was provided by the small damping force in the OFF state; in the ON state, the equivalent damping ratio increased significantly because of the large damping force.

The structure was subjected to a single sine wave excitation with a displacement amplitude of $3 \mathrm{~mm}$. The excitation frequency ranged from 2.0 to $8.0 \mathrm{~Hz}$, which was determined by considering the frequency limit of the exciter and the natural frequency of $2.5 \mathrm{~Hz}$. This corresponded to the frequency ratio $\omega / \omega_{n}$ from 0.8 to 3.2 (Table 2).

To examine the effect of Eq. (3) upon the semi-active vibration suppression performance, the conventional and proposed ON-OFF controls were arranged. Currents of 4 and 0 A were switched according to the sign of $\dot{x}\left(\dot{x}-\dot{x}_{0}\right)$ or $\dot{x}\left(\dot{x}+\dot{x}_{0}\right)$. Their performances were compared with those of passive states with constant currents. Currents of 0 and $4 \mathrm{~A}$ were supplied in the OFF and ON states, respectively. 


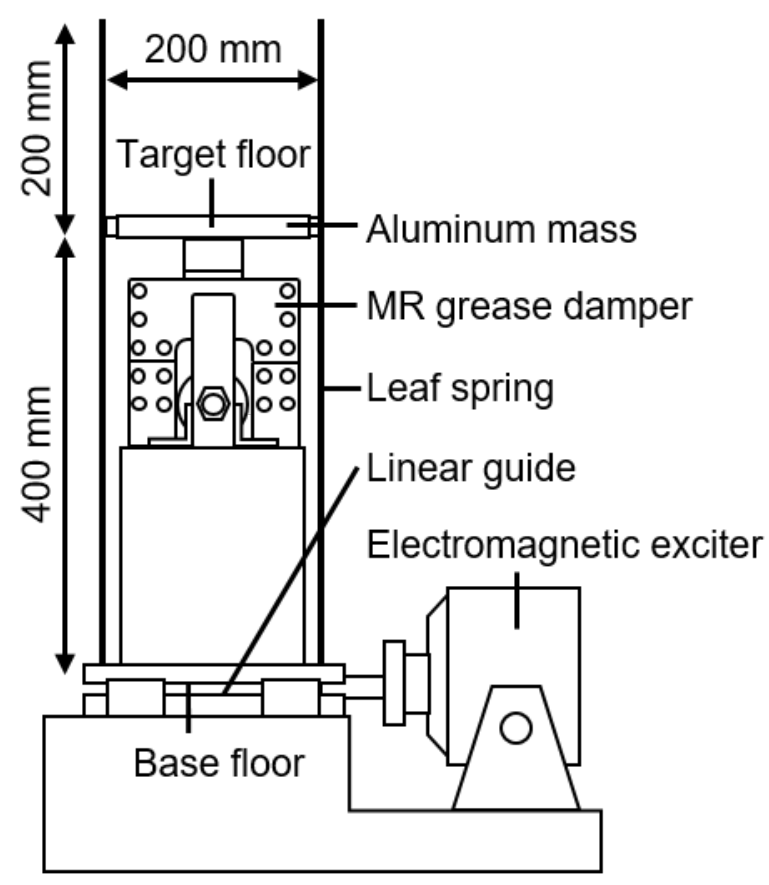

Figure 7. Schematic diagram of the single-degree-of-freedom structure.

Table 2. Conditions for simulations and experimental tests of structural vibration suppression by an MR damper.

\begin{tabular}{ll}
\hline Mass, $m$ & $1.5 \mathrm{~kg}$ \\
Stiffness, $k$ & $370 \mathrm{~N} / \mathrm{m}$ \\
Damping ratio in the OFF state & 0.15 \\
Currents in the passive states & 0 A (OFF state), 4 A (ON state) \\
Current with the conventional ON-OFF control & 4 A if $\dot{x}\left(\dot{x}-\dot{x}_{0}\right)>0,0$ A if $\dot{x}\left(\dot{x}-\dot{x}_{0}\right) \leq 0$ \\
Current with the proposed ON-OFF control & 4 A if $\dot{x}\left(\dot{x}+\dot{x}_{0}\right)>0,0$ A if $\dot{x}\left(\dot{x}+\dot{x}_{0}\right) \leq 0$ \\
Waveform of excitation input, $x_{0}$ & Single sine wave \\
Input amplitude & $3 \mathrm{~mm}$ \\
Input frequency ratio, $\omega / \omega_{n}$ & $0.8-3.2$ \\
\hline
\end{tabular}

\subsection{Simulation methods}

The structure was modeled as a system consisting of a mass, a spring, and a variable damper. If the viscous damping force is included in the external force $F_{\mathrm{MR}}$, the governing equation can be written as follows:

$$
m \ddot{x}+F_{\mathrm{MR}}+k\left(x-x_{0}\right)=0 .
$$

The simulation for obtaining the displacement response was performed in Microsoft Excel using the fourth-order Runge-Kutta integration scheme. Some of the simulation results had been verified through comparison with their exact solutions. The MR damper was installed between the target and base floors; thus, the force $F_{\mathrm{MR}}$ depended upon the supplied current $I$ and the relative velocity between floors, $\dot{x}-\dot{x}_{0}$ :

$$
F_{\mathrm{MR}}=-\left\{a(I)\left|\dot{x}-\dot{x}_{0}\right|+b(I)\right\}\{1-\exp (-t / \tau)\}\left\{1-\exp \left(-\alpha\left|\dot{x}-\dot{x}_{0}\right|\right)\right\} .
$$

The first and third terms are based on the algebraic model, which has been widely used as a simple model for MR dampers (Ruangrassamee et al., 2006; Jiang and Christenson, 2011).

The first force-magnitude term means that the force can be calculated using the supplied current $I$ and relative 
velocity $\dot{x}-\dot{x}_{0}$. The force magnitude can be expressed as a linear function of the absolute value of the relative velocity $\left|\dot{x}-\dot{x}_{0}\right|$, with the gradient and intercept varying with the supplied current $I$. In the first quadrant of the force-velocity diagram, the force magnitude was approximated as a linear function of the velocity $\left|\dot{x}-\dot{x}_{0}\right|$ at each current using the least-squares method (Figure 8). The obtained gradient $a(I)$ and intercept $b(I)$ were approximated as quadratic functions of the current $I$ according to the least-squares method:

$$
a(I)\left|\dot{x}-\dot{x}_{0}\right|+b(I)=\left(a_{2} I^{2}+a_{1} I+a_{0}\right)\left|\dot{x}-\dot{x}_{0}\right|+\left(b_{2} I^{2}+b_{1} I+b_{0}\right) .
$$

Eq. (7) is the detailed form of the first term of Eq. (6). The parameters obtained by the approximations (Table 3) were substituted into Eq. (7) as fixed parameters in the simulation.

To examine the performance under various delay conditions of the damping force, a second term was added as a simple exponential form that included the time constant of the force, $\tau$. A preliminary experiment showed that the response time of the shear-type MR grease damper was $60 \mathrm{~ms}$; thus, the time constant was set to four values: $10,30,60$, and $90 \mathrm{~ms}$.

Finally, the third term, which describes the force-velocity dependence, was added to capture the roll-off at low velocities. The parameter $\alpha$ was determined such that the simulation results corresponded to the experimental ones. In the OFF and ON states, the values were set to 10 and $25 \mathrm{~s} / \mathrm{m}$, respectively.

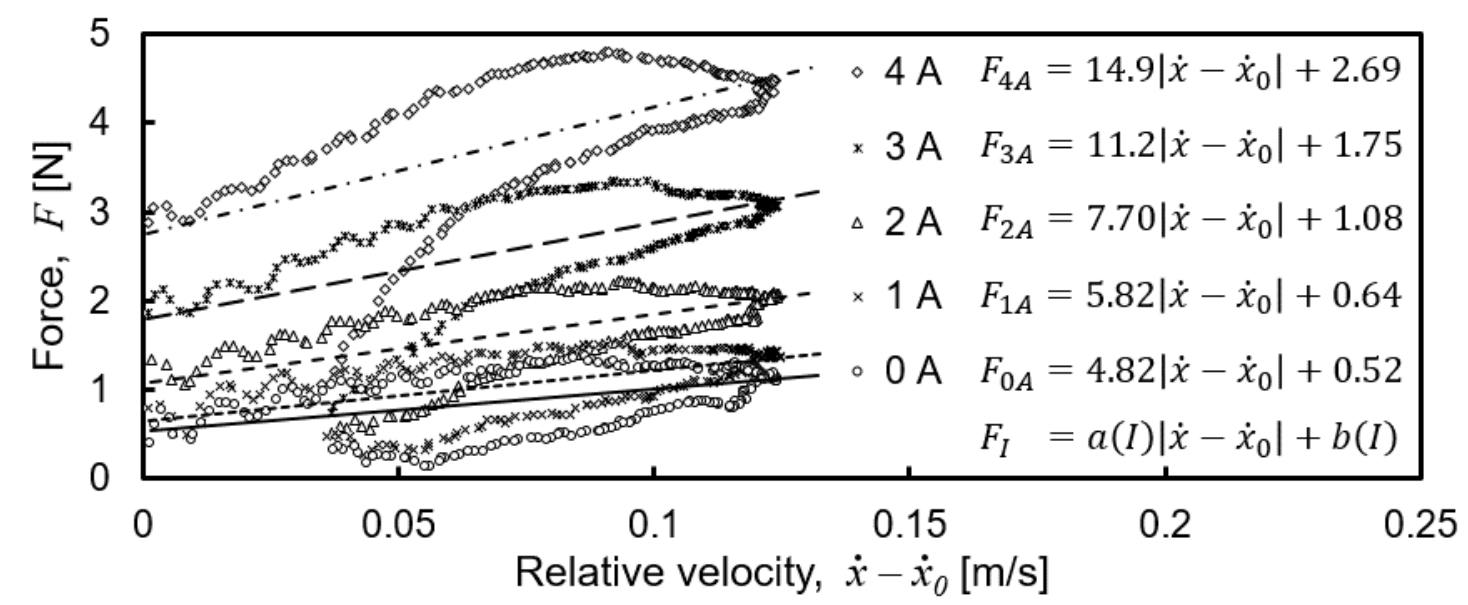

Figure 8. Relationship between the damping force, relative velocity, and current of the shear-type MR grease damper.

Table 3. Parameters in Eq. (7) obtained from the approximation of the gradient and intercept as quadratic functions of the currents.

\begin{tabular}{cccccc}
\hline$a_{2}\left[\mathrm{Ns} / \mathrm{mA}^{2}\right]$ & $a_{1}[\mathrm{Ns} / \mathrm{mA}]$ & $a_{0}[\mathrm{Ns} / \mathrm{m}]$ & $b_{2}\left[\mathrm{~N} / \mathrm{A}^{2}\right]$ & $b_{1}[\mathrm{~N} / \mathrm{A}]$ & $b_{0}[\mathrm{~N}]$ \\
\hline 0.48 & 0.66 & 4.77 & 0.14 & 0.00 & 0.51 \\
\hline
\end{tabular}




\subsection{Experimental methods}

The semi-active structural vibration suppression experiment comprises four procedures: excitation of the base, measurement of the displacements, calculation of the control current, and supply of the calculated current (Figure 9). First, the base floor of the structure was excited by an electromagnetic exciter with a sine wave from a function generator. Then, during the excitation, the displacements on the target and base floors were measured via laser displacement sensors. After that, a controller collected and digitalized the measured displacement data every $0.1 \mathrm{~ms}$ and the control current was calculated following the control strategy that determined the current using the floor velocities. The velocity values were obtained as numerical time derivatives of the measured displacements. They were moving average values of 50 sampling points; thus, the current values were updated every $5 \mathrm{~ms}$. Finally, the current calculated by the controller was supplied from a bipolar power supply to the MR damper. The displacement and current data were recorded on a digital oscilloscope.

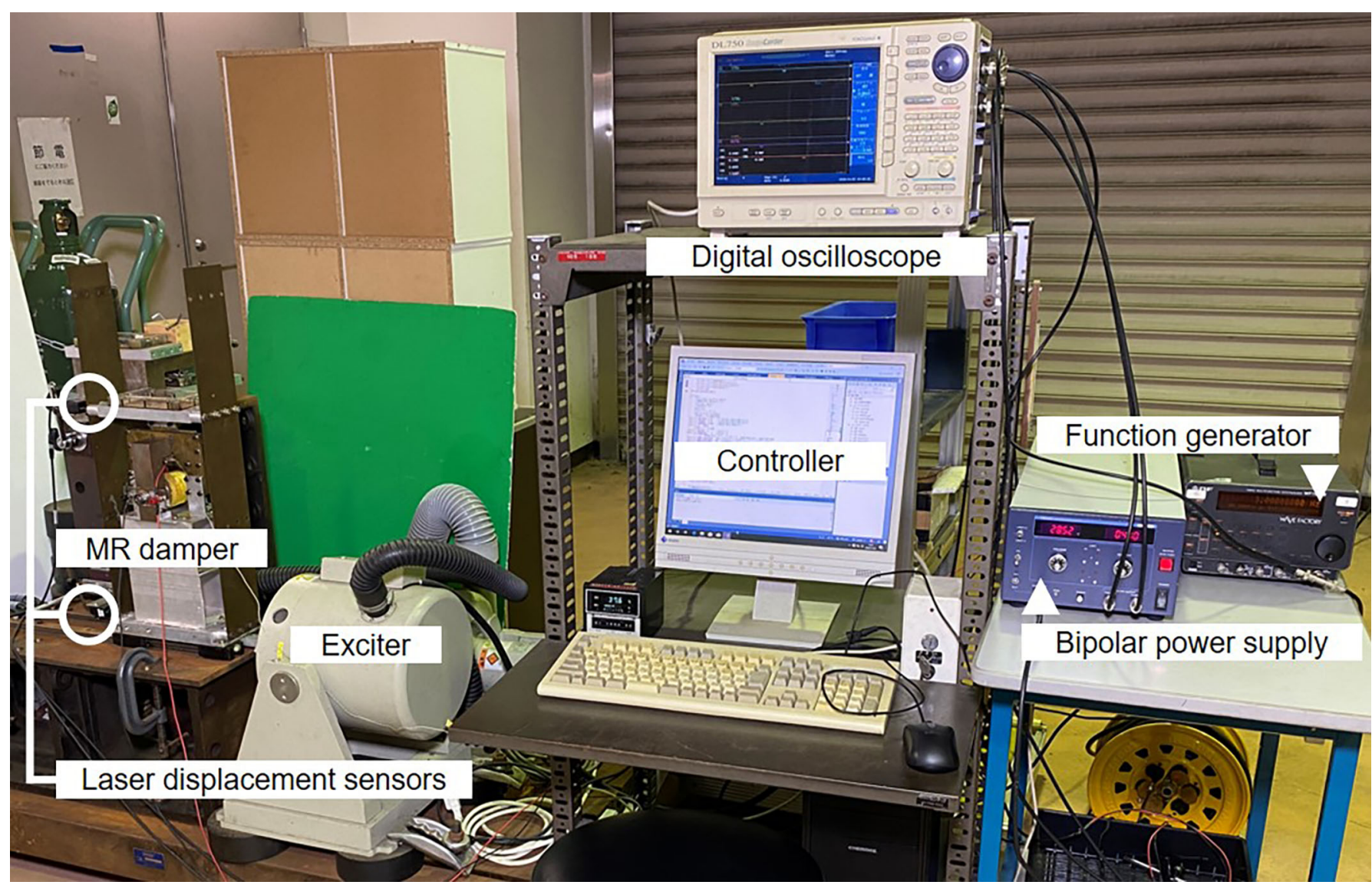

(a) 


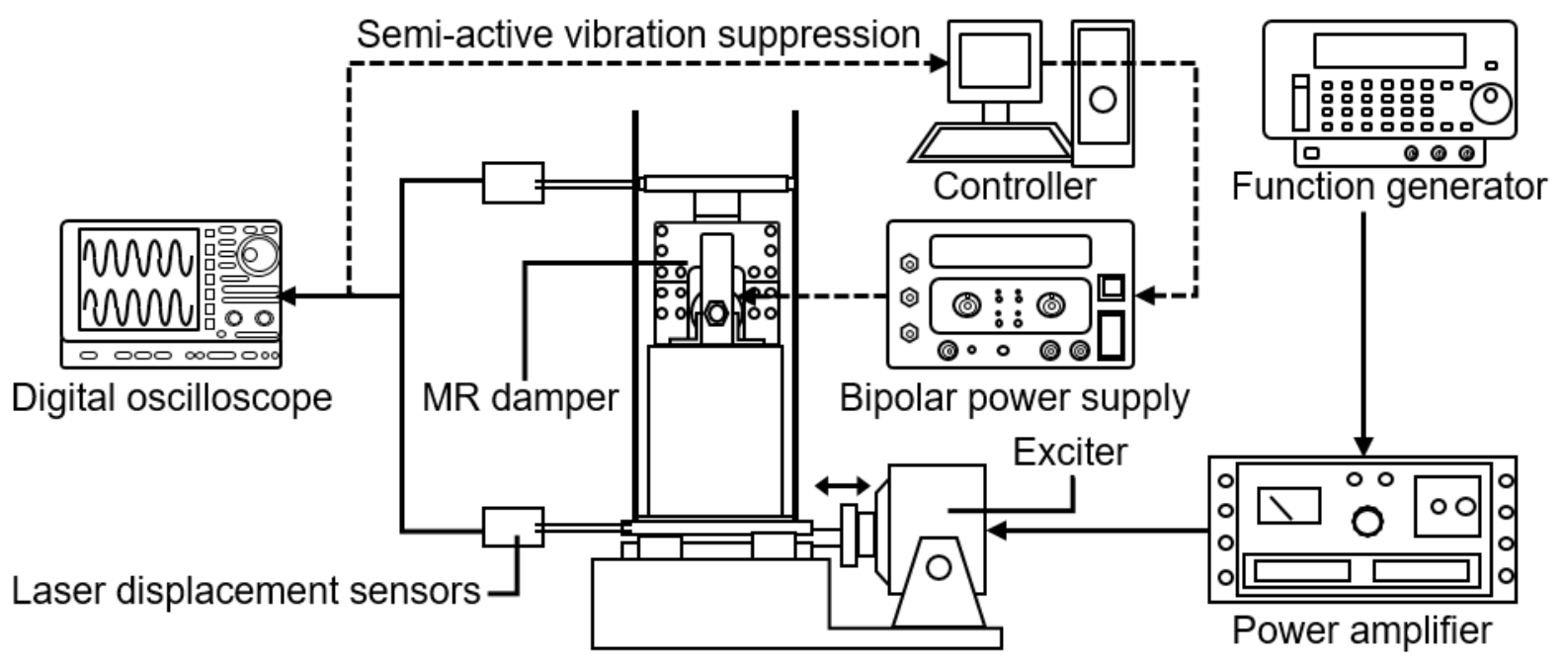

(b)

Figure 9. Setup for the structural vibration suppression experiment: (a) photograph and (b) schematic diagram.

4. Results and Discussion

\subsection{Numerical simulation}

The proposed ON-OFF control using $\dot{x}\left(\dot{x}+\dot{x}_{0}\right)$ provides a high vibration suppression performance, which it maintains even when the response time of the damping force changes from 10 to $90 \mathrm{~ms}$ (Figure 10). Around the natural frequency, the displacement response with the proposed ON-OFF control was always reduced to the same level as the desired ON state, whereas that with the conventional ON-OFF control increased as force delays increased. In the high-frequency range, the proposed $\mathrm{ON}-\mathrm{OFF}$ control provided superior performance compared with the conventional one, regardless of the force response time.

These results demonstrate that the proposed ON-OFF control is more practical, since its performance is maintained even in the presence of force delays in the damper. As mentioned above, in previous studies, the response time typical values of MR dampers have been reported to be several tens of milliseconds. Therefore, when a typical MR damper is applied to the vibration suppression of an actual system, the proposed ON-OFF control can outperform the conventional one over a wide range of frequencies. 


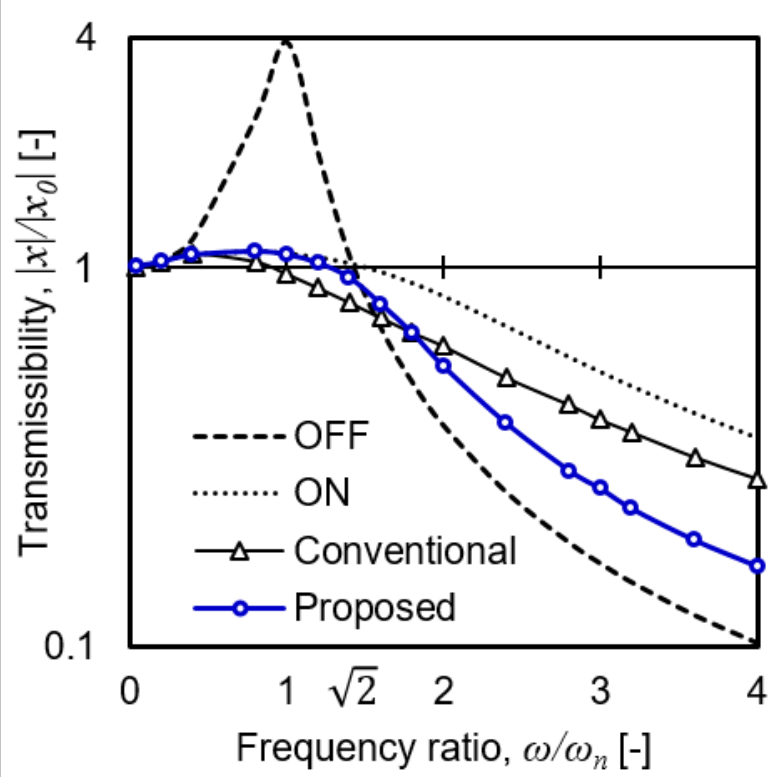

(a)

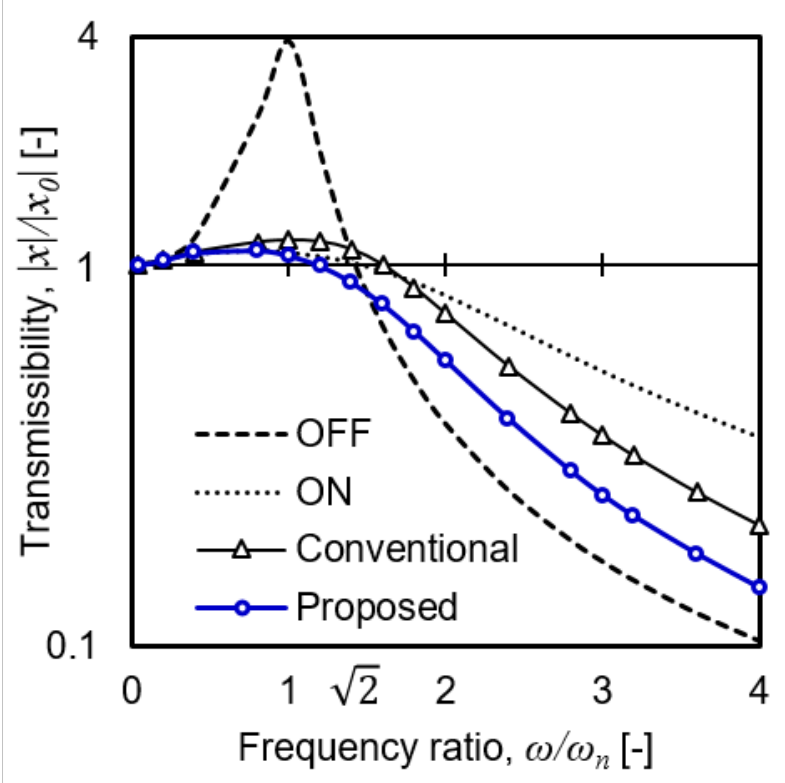

(c)

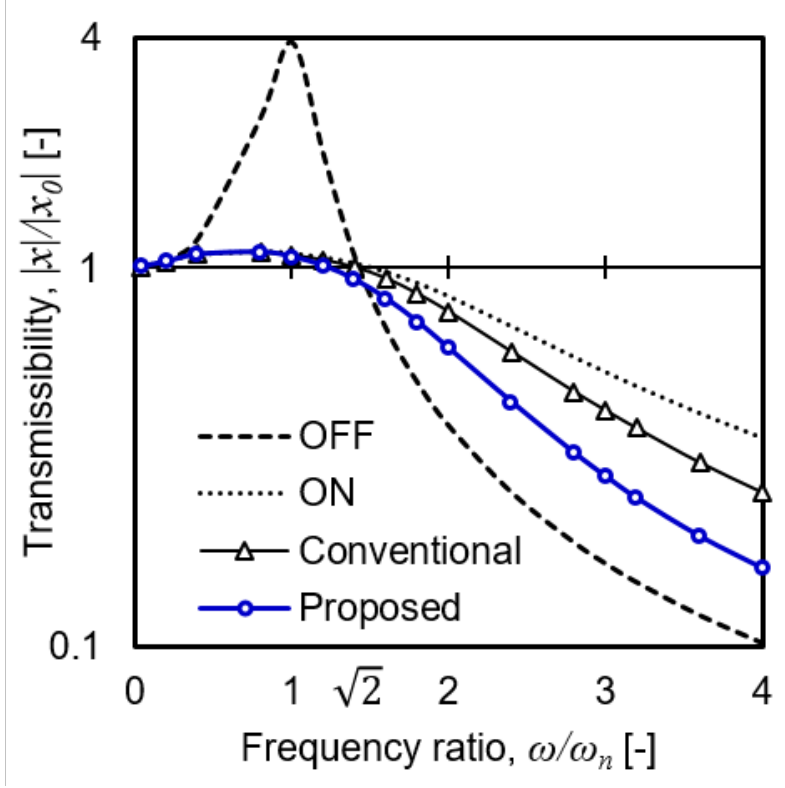

(b)

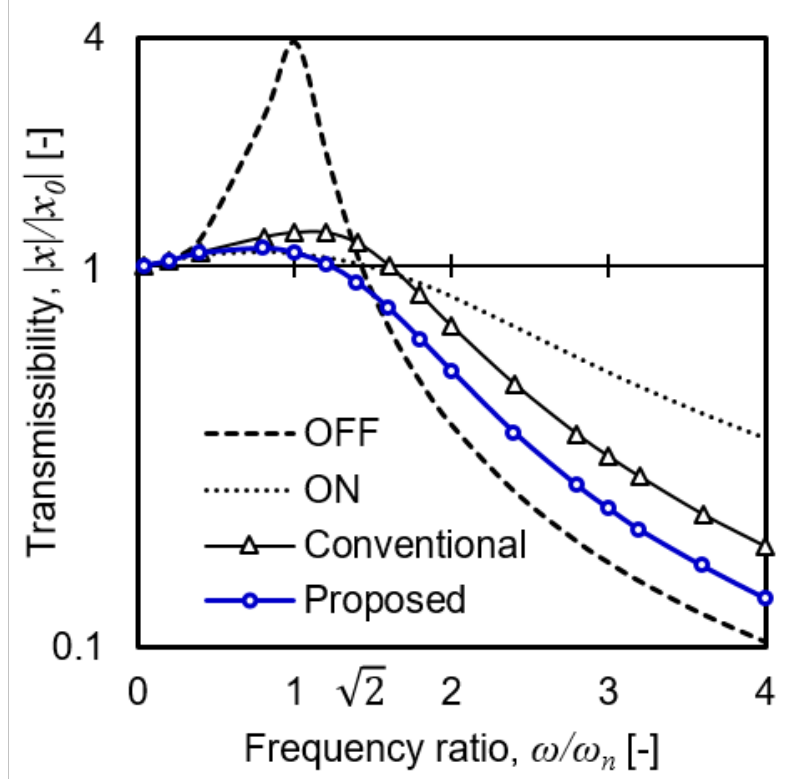

(d)

Figure 10. Simulation results of the frequency response of a structure equipped with an MR damper, having force delays of (a) $10 \mathrm{~ms}$, (b) $30 \mathrm{~ms}$, (c) $60 \mathrm{~ms}$, and (d) $90 \mathrm{~ms}$ upon the application of the conventional and proposed ON-OFF controls. 


\subsection{Experiment}

\subsubsection{Proposed ON-OFF control}

The proposed ON-OFF control using $\dot{x}\left(\dot{x}+\dot{x}_{0}\right)$ provided a superior vibration suppression performance compared with the conventional ON-OFF control using $\dot{x}\left(\dot{x}-\dot{x}_{0}\right)$ over the entire frequency range. Their difference was clear at frequencies higher than $\omega / \omega_{n}=\sqrt{2}$ (Figure 11); according to the simulation results, these results demonstrate that the proposed control strategy is effective not only in the simulation but also in the experiment with the delay in damping force and that its advantage over the conventional strategy becomes significant as frequency increases.

Around the natural frequency, the displacement response of the proposed ON-OFF control was reduced to the same level as in the ON state. The desired performance was achieved by the proposed control. The ratio of the ON time reached $97 \%$ due to $\dot{x}\left(\dot{x}+\dot{x}_{0}\right)$ being positive for almost the entire period at $\omega / \omega_{n}=1.0$ (Figure 12 (a)). In the mid-frequency range, the proposed $\mathrm{ON}-\mathrm{OFF}$ control outperformed the conventional one in terms of displacement response reduction, although the desired OFF performance was not achieved. The ON time at $\omega / \omega_{n}=2.0$ reached $67 \%$ (Figure 12 (b)); thus, it should be reduced for higher performance. In the high-frequency range, the proposed $\mathrm{ON}-\mathrm{OFF}$ control performed well and significantly improved the performance of the conventional ON-OFF control. At $\omega / \omega_{n}=3.0$, the ratio of the ON time was reduced to only $25 \%$ because of $\dot{x}\left(\dot{x}+\dot{x}_{0}\right)$ being negative for a long period of time (Figure 12 (c)). Although the waveforms of $\dot{x}\left(\dot{x}+\dot{x}_{0}\right)$ were distorted due to the control current effect and the velocity calculation of numerical time derivatives and moving average using 50 sampling points, it did not affect the periodic ON/OFF switching of Eq. (3) and the vibration suppression performance.

These results demonstrate that the ON-OFF control using $\dot{x}\left(\dot{x}+\dot{x}_{0}\right)$ can perform as expected according to the theory of forced vibration with base excitation, especially in the vicinity of the natural frequency and in the high-frequency range. For $0 \leq \omega / \omega_{n} \leq \sqrt{2}$, the velocity-amplitude ratio $\left|\dot{x} / \dot{x}_{0}\right|$ is 1 or more, regardless of the damping ratio; thus, $\dot{x}\left(\dot{x}+\dot{x}_{0}\right)$ is always 0 or more, as shown by the desired switching (Table 1). This allows the proposed $\mathrm{ON}-\mathrm{OFF}$ control to perform as the $\mathrm{ON}$ state for a long time and to definitely achieve the desired vibration suppression performance. In the range of $\omega / \omega_{n}>\sqrt{2}$, conversely, the performance is degraded by the constant large current of $4 \mathrm{~A}$ that is supplied when $\dot{x}\left(\dot{x}+\dot{x}_{0}\right)>0$ is achieved. When the control strategy with $\dot{x}\left(\dot{x}+\dot{x}_{0}\right)$ was proposed, the damping ratio was assumed to be so low that $\dot{x}$ and $\dot{x}_{0}$ were in completely opposite phases. However, in the experiment, the damping ratio becomes higher than assumed because of the large damping force exerted at $4 \mathrm{~A}$. It is necessary to adjust the magnitude of the current according to the vibration to improve the performance of the proposed ON-OFF control in the range of $\omega / \omega_{n}>\sqrt{2}$, especially in the mid-frequency range around $\omega / \omega_{n}=2.0$. 


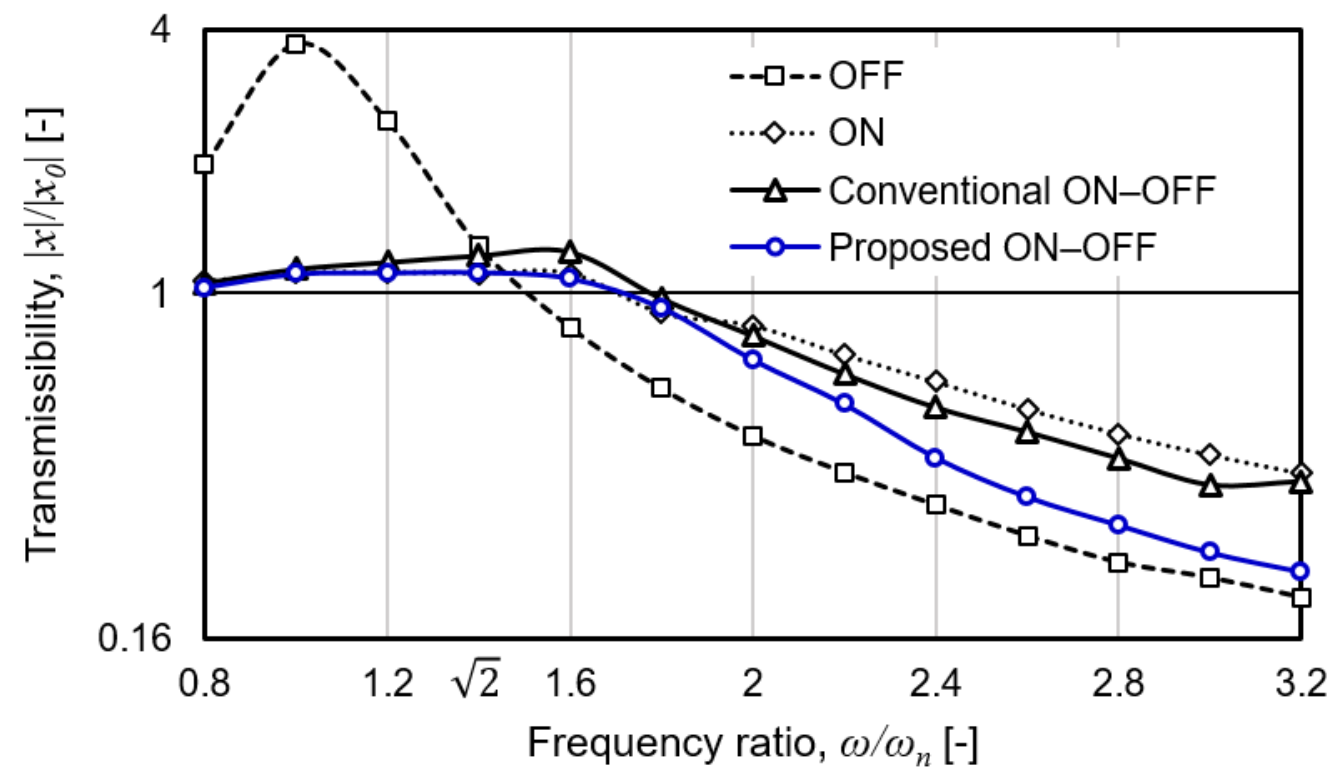

Figure 11. Experimental frequency response of the structure equipped with an MR damper under sine wave excitation upon applying the conventional and proposed ON-OFF controls. 

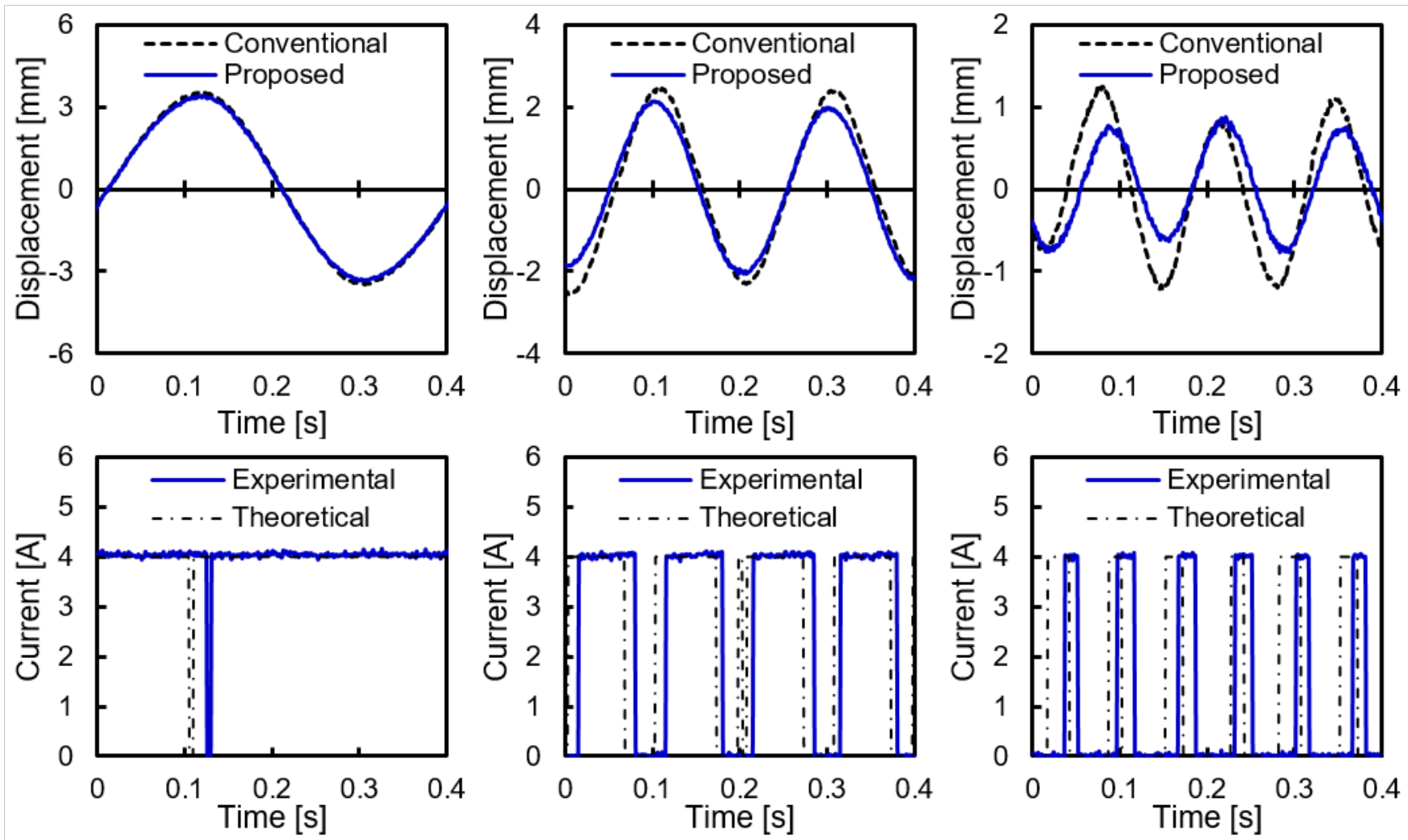

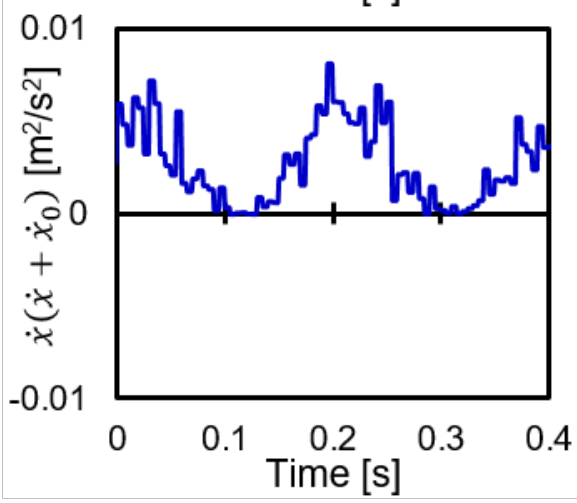

(a)

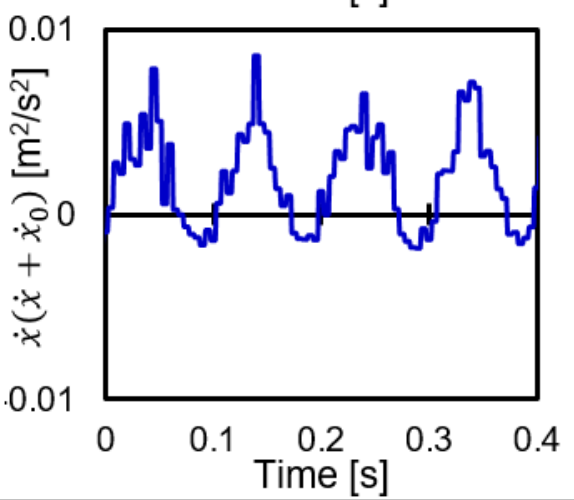

(b)

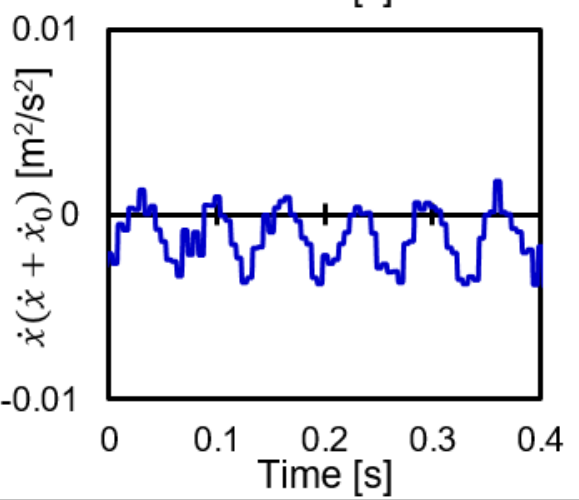

(c)

Figure 12. Time series of the displacement of the target floor (upper), the supplied current (middle), and the condition function $\dot{x}\left(\dot{x}+\dot{x}_{0}\right)$ (lower) with the proposed ON-OFF control at (a) $\omega / \omega_{n}=1.0$, (b) $\omega / \omega_{n}=2.0$, and (c) $\omega / \omega_{n}=3.0$. The displacements are compared with those with the conventional ON-OFF control. 


\subsubsection{Proposed velocity-proportional control}

This section presents a novel velocity-proportional control scheme by modifying the proposed ON-OFF control to improve its performance in the mid- and high-frequency range while maintaining a high performance in the vicinity of the natural frequency. The proposed velocity-proportional control offers a damping force proportional to the absolute velocity, $F_{c}$, when $\dot{x}\left(\dot{x}+\dot{x}_{0}\right)>0$ is achieved:

$$
F= \begin{cases}F_{c}, & \dot{x}\left(\dot{x}+\dot{x}_{0}\right)>0 \\ F_{O F F}, & \dot{x}\left(\dot{x}+\dot{x}_{0}\right) \leq 0\end{cases}
$$

This strategy utilizes the velocity-proportional damping force required in the skyhook damper scheme in combination with $\dot{x}\left(\dot{x}+\dot{x}_{0}\right)$. The aim of this control strategy is ON/OFF switching at $\omega / \omega_{n}=\sqrt{2}$, rather than emulating the forces of the skyhook damper; thus, the name "skyhook" is not included. To achieve the required force $F_{c}$, the supplied current was continuously varied along with the target and base velocities. The damping coefficient $c$ was updated using the following equation:

$$
c=c_{p}\left|\frac{\dot{x}}{\dot{x}-\dot{x}_{0}}\right|,
$$

where $c_{p}$ denotes the proportionality constant of $35 \mathrm{Ns} / \mathrm{m}$, which was most effective for experimental response reduction. When the relative velocity was zero, the control current was switched to $0 \mathrm{~A}$. To facilitate the calculation of Eq. (9), the RMS values of velocity may be applicable though the moving average values of 50 sampling points were used in this study. By controlling the current according to the velocities to achieve Eq. (9), a damping force proportional to the absolute velocity can be exerted on the suppression target:

$$
\left|F_{c}\right| \cong c\left|\dot{x}-\dot{x}_{0}\right|=c_{p}\left|\frac{\dot{x}}{\dot{x}-\dot{x}_{0}}\right|\left|\dot{x}-\dot{x}_{0}\right|=c_{p}|\dot{x}| .
$$

Although an update cannot be achieved in conventional studies because of their low dynamic ranges, it was realized in this study by taking full advantage of the high dynamic range of the shear-type MR grease damper.

The proposed velocity-proportional control provided both the desired ON performance around the natural frequency and the desired OFF performance in the high-frequency range. A clearly improved performance was achieved compared with that of the ON-OFF control proposed in the previous section, especially in the midfrequency range of $1.8 \leq \omega / \omega_{n} \leq 2.8$ (Figure 13). Around the natural frequency, the displacement response with the proposed velocity-proportional control was reduced to the same level as in the desired ON state and with the proposed ON-OFF control. The variable current supplied at $\omega / \omega_{n}=1.0$ was sufficient to suppress the vibration successfully, although it did not coincide with the constant current of $4 \mathrm{~A}$ in the fully ON state (Figure 14 (a)). In the mid- and high-frequency range, the proposed velocity-proportional control provided the desired OFF performance in two steps. First, the continuously controlled current allowed the damping force to approach that in the OFF state; then, since the reduced force lowered the equivalent damping ratio of the target system, the assumption of $\dot{x}\left(\dot{x}+\dot{x}_{0}\right) \leq 0$ in the range of $\omega / \omega_{n}>\sqrt{2}$ was achieved for a longer time. At $\omega / \omega_{n}=2.0$, the ratio of the ON time was lowered from $67 \%$ to $36 \%$ by reducing the maximum current from 4.0 to $2.7 \mathrm{~A}$ (Figure 14 (b)). Similarly, at $\omega / \omega_{n}=3.0$, the ratio of the ON time was lowered from $25 \%$ to $17 \%$ by reducing the maximum current from 4.0 to 2.2 A (Figure 14 (c)). These results suggest that the desired vibration suppression 
performance can be achieved by applying $\dot{x}\left(\dot{x}+\dot{x}_{0}\right)$ in combination with the velocity-proportional control.

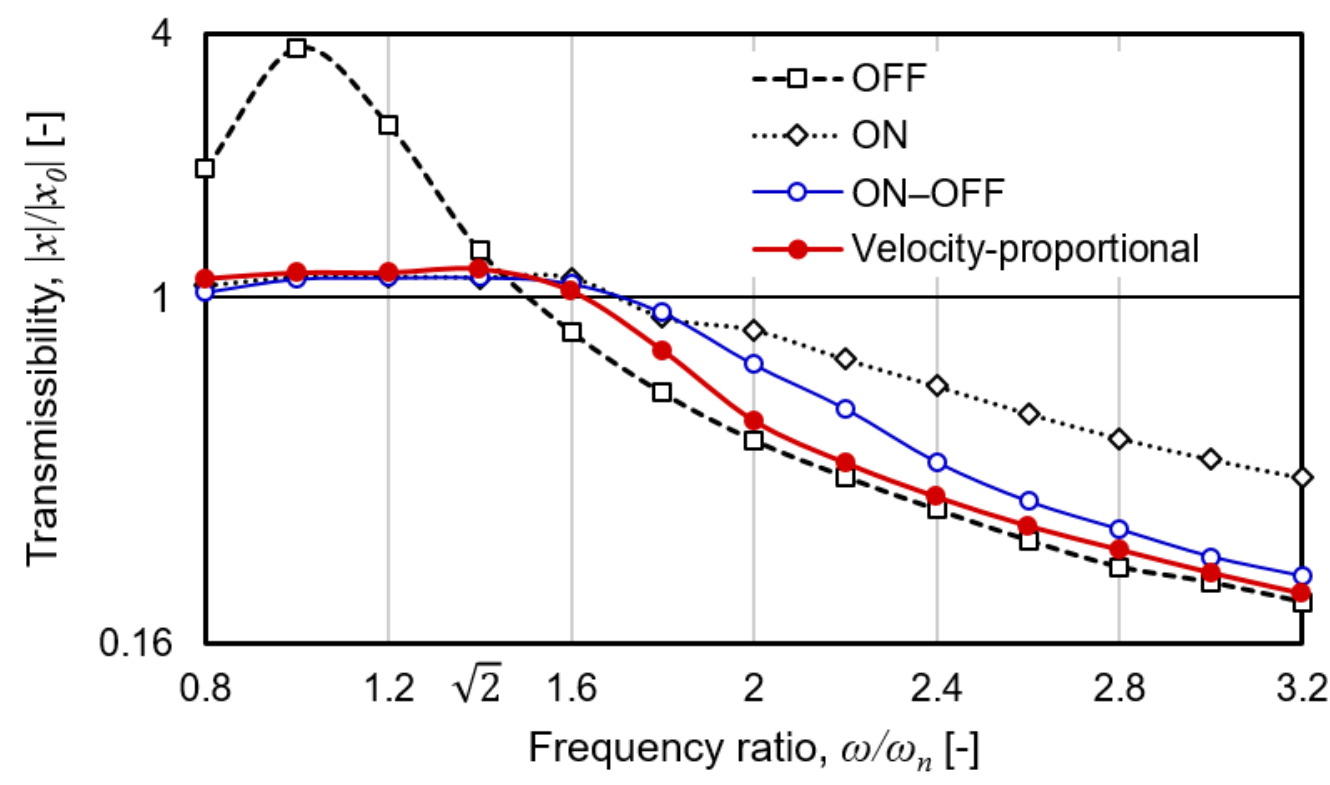

Figure 13. Experimental results of the frequency response of the structure equipped with an MR damper under sine wave excitation upon applying the proposed $\mathrm{ON}-\mathrm{OFF}$ and velocity-proportional control. 

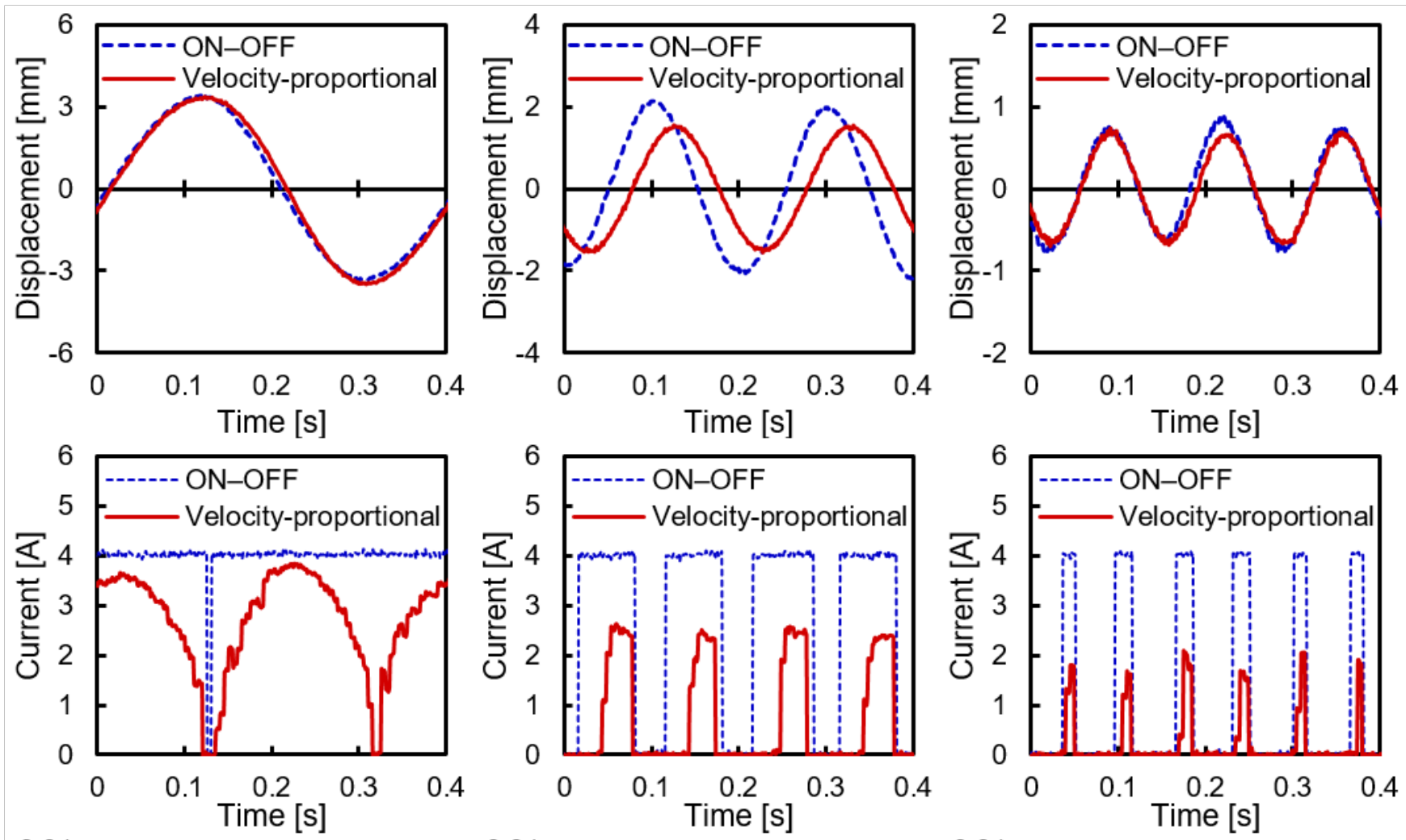

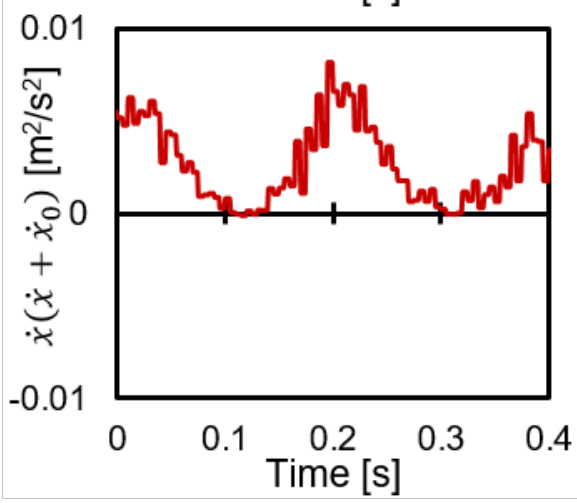

(a)

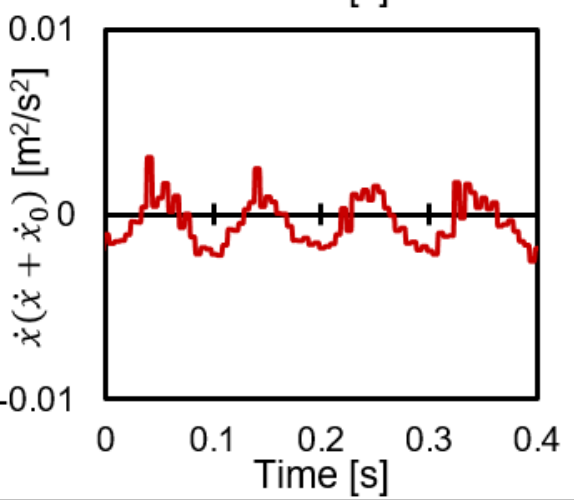

(b)

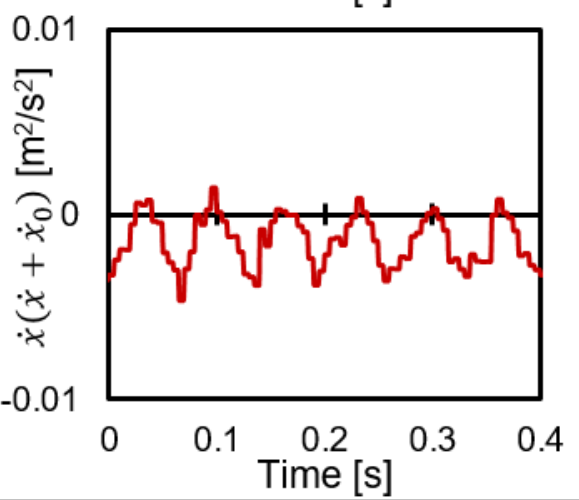

(c)

Figure 14. Time series of the displacement of the target floor (upper), the supplied current (middle), and the condition function $\dot{x}\left(\dot{x}+\dot{x}_{0}\right)$ (lower) with the proposed velocity-proportional control at (a) $\omega / \omega_{n}=1.0$, (b) $\omega / \omega_{n}=2.0$, and (c) $\omega / \omega_{n}=3.0$. The displacements and currents are compared with those with the proposed ON-OFF control. 
5. Conclusion

In this study, simple control strategies using a novel condition function $\dot{x}\left(\dot{x}+\dot{x}_{0}\right)$ were proposed for semiactive vibration suppression of single-degree-of-freedom systems and formulated based on the theory of forced vibration with base excitation. Moreover, its effect upon the semi-active vibration suppression performance was investigated using numerical simulations and experiments on a small structure equipped with an MR damper. Our formulation elucidates that the proposed control strategy can act as an ON state around the natural frequency and as an OFF state in the high-frequency range under the assumption of a low damping ratio of the target system. The simulation and experimental results demonstrate that ON-OFF control with $\dot{x}\left(\dot{x}+\dot{x}_{0}\right)$ offers improved vibration suppression performance compared with that of the conventional control, especially in the high-frequency range. Furthermore, the velocity-proportional control with $\dot{x}\left(\dot{x}+\dot{x}_{0}\right)$ achieves the desired performance by exerting small damping forces upon the suppression target with a continuously controlled current in the mid- to high-frequency range. These results indicate that the proposed simple control strategy can be applied to practical single-degree-offreedom systems, since it has been shown by both simulation and experiment to provide the desired vibration suppression performance.

\section{References}

Casciati F, Rodellar J and Yildirim U (2012) Active and semi-active control of structures - theory and applications: A review of recent advances. Journal of Intelligent Material Systems and Structures 23(11): 1181-1195.

Cha YJ, Agrawal AK and Dyke SJ (2013) Time delay effects on large-scale MR damper based semi-active control strategies. Smart Materials and Structures 22(1): 015011.

Choi SB, Li WH, Yu M et al. (2016) State of the art of control schemes for smart systems featuring magneto-rheological materials. Smart Materials and Structures, 25(4): 043001.

Dyke SJ, Spencer Jr. BF, Sain MK, et al. (1996) Modeling and control of magnetorheological dampers for seismic response reduction. Smart Materials and Structures 5(5): 565.

Dyke SJ, Spencer Jr. BF, Sain MK, et al. (1998) An experimental study of MR dampers for seismic protection. Smart Materials and Structures 7(5): 693.

Jiang Z and Christenson R (2011) A comparison of $200 \mathrm{kN}$ magneto-rheological damper models for use in real-time hybrid simulation pretesting. Smart Materials and Structures 20(6): 065011.

Karnopp D, Crosby MJ and Harwood RA (1974) Vibration control using semi-active force generators. Transactions of the American Society of Mechanical Engineers, Journal of Manufacturing Science and Engineering 96(2): 619-626.

Liu Y, Waters TP and Brennan MJ (2005) A comparison of semi-active damping control strategies for vibration isolation of harmonic disturbances. Journal of Sound and Vibration 280(1-2): 21-39.

Potter JN, Neild SA and Wagg DJ (2010) Generalisation and optimisation of semi-active, on-off switching controllers for single degree-of-freedom systems. Journal of Sound and Vibration 329(13): 2450-2462.

Qin Y, Zhao F, Wang Z, et al. (2017) Comprehensive analysis for influence of controllable damper time delay on semiactive suspension control strategies. Transactions of the American Society of Mechanical Engineers. Journal of Vibration and Acoustics 139(3): 031006. 
Ruangrassamee A, Srisamai W and Lukkunaprasit P (2006) Response mitigation of the base isolated benchmark building by semi-active control with the viscous-plus-variable-friction damping force algorithm. Structural Control and Health Monitoring 13(2-3): 809-822.

Saaed TE, Nikolakopoulos G, Jonasson JE, et al. (2013) A state-of-the-art review of structural control systems. Journal of Vibration and Control. 21(5): 919-937.

Shiraishi T and Sugiyama T (2015) Development of a shear type controllable damper with magnetorheological grease. Bulletin of the Japan Society of Mechanical Engineers, Mechanical Engineering Journal 2(3).

Strecker Z, Mazurek I, Roupec J, et al. (2015a) Influence of MR damper response time on semiactive suspension control efficiency. Meccanica 50(8): 1949-1959.

Strecker Z, Roupec J, Mazurek I, et al. (2015b) Design of magnetorheological damper with short time response. Journal of Intelligent Material Systems and Structures 26(14): 1951-1958.

Strecker Z, Roupec J, Mazurek I, et al. (2018) Influence of response time of magnetorheological valve in Skyhook controlled three-parameter damping system, Advances in Mechanical Engineering 10(11): 1-8.

Symas MD and Constantinou MC (1999) Semi-active control systems for seismic protection of structures: A state-ofart review. Engineering Structures 21(6): 469-487.

Yang G, Spencer Jr. BF, Carlson JD, et al. (2002) Large-scale MR fluid dampers: modeling and dynamic performance considerations. Engineering Structures 24(3): 309-323.

Yao GZ, Yap FF, Chen G, et al. (2002) MR damper and its application for semi-active control of vehicle suspension system. Mechatronics 12(7): 963-973.

Yoon DS, Kim GW and Choi SB (2021) Response time of magnetorheological dampers to current inputs in a semiactive suspension system: modeling, control and sensitivity analysis. Mechanical Systems and Signal Processing 146(1): 106999. 